\title{
Cyclooxygenase-1-selective inhibition prolongs gestation in mice without adverse effects on the ductus arteriosus
}

\author{
Charles D. Loftin, Darshini B. Trivedi, and Robert Langenbach \\ Laboratory of Environmental Carcinogenesis and Mutagenesis, National Institutes of Health, \\ National Institute of Environmental Health Sciences, Research Triangle Park, North Carolina, USA
}

\begin{abstract}
Preterm delivery is the leading cause of neonatal mortality and contributes significantly to infant morbidity. Classical cyclooxygenase (COX) inhibitors, such as indomethacin, which inhibit both COX-1 and COX-2, are effective for delaying premature labor, but their use is limited by serious complications to the fetus and neonate, including adverse effects on the ductus arteriosus (DA). Using isoform-selective inhibitors, we characterized the roles of the COX isoforms in the initiation of labor and the regulation of fetal and neonatal DA closure in mice. Chronic inhibition of COX-2 during pregnancy (gestation days 15-18) significantly increased neonatal mortality by preventing closure of the DA after birth, whereas acute COX-2 inhibition near the end of term (gestation day 18) constricted the fetal DA. In contrast, the inhibition of COX-1 during pregnancy lacked these prenatal and postnatal adverse effects on the DA and effectively delayed the initiation of full-term labor and LPSinduced preterm labor. These findings suggest that premature fetal DA closure or neonatal patent DA observed following indomethacin tocolysis in women may result from the inhibition of COX-2. Therefore, COX-1-selective inhibitors may provide effective treatment to delay preterm labor with fewer adverse effects on fetal or neonatal health than nonselective or COX-2-selective inhibitors.
\end{abstract}

J. Clin. Invest. 110:549-557 (2002). doi:10.1172/JCI200214924.

\section{Introduction}

Preterm delivery is the leading cause of perinatal mortality and contributes to delayed physical and cognitive development in children. Although a significant number of preterm cases would benefit from delaying labor, there is considerable debate on the safety and efficacy of currently available medications for the maintenance of tocolysis (1-3). Prostaglandin (PG) synthase (cyclooxygenase, or COX) inhibitors, also known as nonsteroidal anti-inflammatory drugs (NSAIDs), represent one of the several classes of tocolytics effective for delaying labor in the short term, but their usefulness is limited, in part, by adverse effects on the fetus $(4,5)$. One of the major adverse effects of NSAIDs is on the ductus arteriosus (DA). The DA is a muscular artery that connects the main pulmonary artery and the aorta. PGs in the fetal circulation are thought to maintain dilation of the DA in utero. The dilated DA in the fetus diverts the pulmonary circulation away from the high-resistance vascular beds of the uninflated lungs.

Received for publication December 20, 2001, and accepted in revised form June 25, 2002.

Address correspondence to: Robert Langenbach, National Institute of Environmental Health Sciences, Mail Drop C4-09, PO Box 12233, Research Triangle Park, North Carolina 27709, USA. Phone: (919) 541-7558; Fax: (919) 541-1460;

E-mail: langenbach@niehs.nih.gov.

Conflict of interest: No conflict of interest has been declared.

Nonstandard abbreviations used: prostaglandin (PG);

cyclooxygenase (COX); nonsteroidal anti-inflammatory drugs (NSAIDs); ductus arteriosus (DA).
After birth, the transition to pulmonary respiration is accompanied by the constriction and functional closure of the DA. The failure of the DA to close after birth, termed patent DA, is a neonatal complication often associated with premature births.

Indomethacin, the dual COX isoform inhibitor most commonly used to delay premature labor, is associated with two paradoxical effects on the DA. Indomethacin is reported to induce constriction of the fetal DA in as many as $50 \%$ of cases following maternal administration (5-7). In both humans and rodents, acute maternal administration of indomethacin induces constriction of the fetal DA by inhibiting the synthesis of PGs important for DA dilation in utero $(6,8,9)$. In utero constriction of the DA impedes blood flow through the vessel and may result in pulmonary hypertension or heart failure in the fetus $(5,10)$. Alternatively, maternal administration of indomethacin during pregnancy can also increase the risk for development of patent DA in infants after birth $(4,11,12)$. Patent DA adversely affects neonatal health by contributing to respiratory distress, congestive heart failure, and conditions resulting from tissue ischemia (13).

The COX inhibitors that have been used for tocolysis, such as indomethacin, inhibit activity of the two known isoforms, COX-1 and COX-2. Recently, medications selective for inhibiting the COX-2 isoform have been suggested as novel tocolytics with fewer adverse effects than result from dual COX isoform inhibitors $(2,14,15)$. However, we recently reported that in mice, the expression of COX-2 was essential for 
eliminating a patent DA phenotype (16). Herein, we show that in mice, selective inhibition of COX-2 results in fetal DA constriction or postnatal DA paten$\mathrm{cy}$, depending on the dosing regimen, and does not affect the normal onset of labor. Furthermore, selective inhibition of COX-1 does not cause fetal DA constriction or neonatal patent DA and is effective for delaying the initiation of labor.

\section{Methods}

Mice. Mice with targeted disruptions in the genes encoding either COX-1 (17) or COX-2 (18) were maintained on an outbred genetic background of C57BL/6J and $129 / \mathrm{Ola}$. Wild-type mice were generated from the COX-deficient lines to maintain a similar genetic background. The morning after pairing was designated as gestation day 0 upon detection of a copulation plug. COX-1 heterozygous- or homozygous-deficient mice were generated from COX-1 heterozygous-deficient females mated to COX-1 homozygous-deficient males. Mice deficient in COX-2 were generated from COX-2 heterozygous-deficient females mated to COX-2 homozygous-deficient males. $\mathrm{COX}-1^{-/-} / \mathrm{COX}-2^{-/-}$ neonates were generated from $\mathrm{COX}-1^{-/-} / \mathrm{COX}-2^{+/-}$ females mated to $\mathrm{COX}-1^{-/-} / \mathrm{COX}-2^{+/-}$males. COX-deficient mice were genotyped by PCR as previously described (16). All experiments were conducted in accordance with the National Institute of Environmental Health Sciences Animal Care and Use Program. Pathology. Histological analysis was performed on formalin-fixed, paraffin-embedded tissue. Decapitated torsos were transected below the rib cage before formalin fixing. Upper and lower torsos were processed, embedded in paraffin, and serial-sectioned. Sections of kidney, liver, pancreas, spleen, thymus, intestine, stomach, heart, and lungs were obtained for pathological analysis. DA analysis was performed on transverse sections of the upper thorax. The entire length of the DA from the descending aorta to the bifurcation of the pulmonary arteries was serial-sectioned. The photos shown in the figures are representative of the sections most central to the DA lumen (stained with hematoxylin and eosin).

Neonatal DA analysis. Patent DA incidence (no. mice with patent DA/no. mice born) following chronic isoform-selective COX inhibition during pregnancy was determined in neonates 5 hours after birth. Chronic isoform-selective COX inhibition during pregnancy was achieved by maternal administration on gestation days $15-18$. In rodents, the plasma $t_{1 / 2}$ of SC-236 is 117 hours (19), which allowed for dosing once per day $(20$ $\mathrm{mg} / \mathrm{kg}$ oral gavage) on gestation days 15 and 17 , followed by $50 \mathrm{mg} / \mathrm{kg}$ on gestation day 18 . Because of the shorter $t_{1 / 2}$, SC-560 and celecoxib (19) were administered ( $50 \mathrm{mg} / \mathrm{kg}$ oral gavage) twice a day on gestation days 15-17 and once on gestation day 18. Four hours after treatment on gestation day 18, litters were delivered by cesarean section and neonates were sacrificed by decapitation 5 hours later for DA analysis. DA closure following vehicle or COX-2 inhibitor (SC-236) administration during pregnancy was also determined in neonates after natural birth. The method of delivery (cesarean section or natural birth) did not significantly alter patent DA incidence in vehicle- or SC-236-treated neonates. Litters born by natural delivery were monitored with digital cameras to determine the exact time of birth. Significant differences in patent DA incidence between vehicle and COX inhibitor treatments were determined by $\chi^{2}$ analysis.

Fetal DA analysis. To study the effects of an acute dose of COX inhibitors on the DNA of near-term fetuses, indomethacin (Cayman Chemical Co., Ann Arbor, Michigan, USA), SC-560, celecoxib (kindly provided by Rachel Slade, Myriad Pharmaceuticals Inc., Salt Lake City, Utah, USA), and SC-236 (Calbiochem-Novabiochem Corp., La Jolla, California, USA) were each administered on gestation day 18 at $20 \mathrm{mg} / \mathrm{kg}$ by oral gavage with $5 \%$ gum arabic to pregnant mice fasted overnight. Four hours after dosing, the fetuses were delivered by cesarean section and sacrificed by decapitation for DA analysis. Significant differences between the incidence of fetal DA constriction following acute treatments with vehicle or COX inhibitors were determined by $\chi^{2}$ analysis.

To study the effects of chronic COX-2 inhibition on the DA in near-term fetuses, SC-236 was administered $(20 \mathrm{mg} / \mathrm{kg}$ ) on gestation days 15 and 17, followed by 20 or $50 \mathrm{mg} / \mathrm{kg}$ on gestation day 18 . Fetuses were delivered by cesarean section and sacrificed by decapitation for histological analysis of the DA at 4 hours after the initial dose administered on gestation day 15,4 hours after the second dose given on gestation day 17 , or 4 hours after a final dose administered on gestation day 18 .

Neonatal mortality. Pups were monitored for COX-2 inhibitor-induced mortality during the first 48 hours after birth. Pregnant mice were treated with SC-236 (20 $\mathrm{mg} / \mathrm{kg}$ oral gavage) on gestation days 15 and 17 , followed by $50 \mathrm{mg} / \mathrm{kg}$ on gestation day 18 . The pups that died were collected for pathological analysis. Pups that survived beyond 48 hours were sacrificed 52 hours after birth for pathological analysis.

Delayed full-term and preterm labor. The potential of isoform-selective COX inhibitors to delay the initiation of full-term and preterm labor was determined in wildtype mice. For determining isoform-selective COX inhibitor effects on normal gestation length, SC-560 or celecoxib (50 mg/kg oral gavage) was administered twice a day on gestation days $15-18$. SC-236 $(20 \mathrm{mg} / \mathrm{kg}$ oral gavage) was administered on gestation days 15 and 17 , followed by $50 \mathrm{mg} / \mathrm{kg}$ on gestation day 18 . The LPSinduced mouse model of preterm labor $(20,21)$ was used to determine the effect of COX-1-selective inhibition on preterm labor. Labor was induced by a single intraperitoneal injection of bacterial LPS $(1 \mathrm{mg} / \mathrm{kg}$ Escherichia coli 0111:B4; Sigma-Aldrich, St. Louis, Missouri, USA) on gestation day 15.2 . SC-560 (50 mg/kg oral gavage) or vehicle administration was initiated 24 hours prior to LPS injection and continued twice a day 

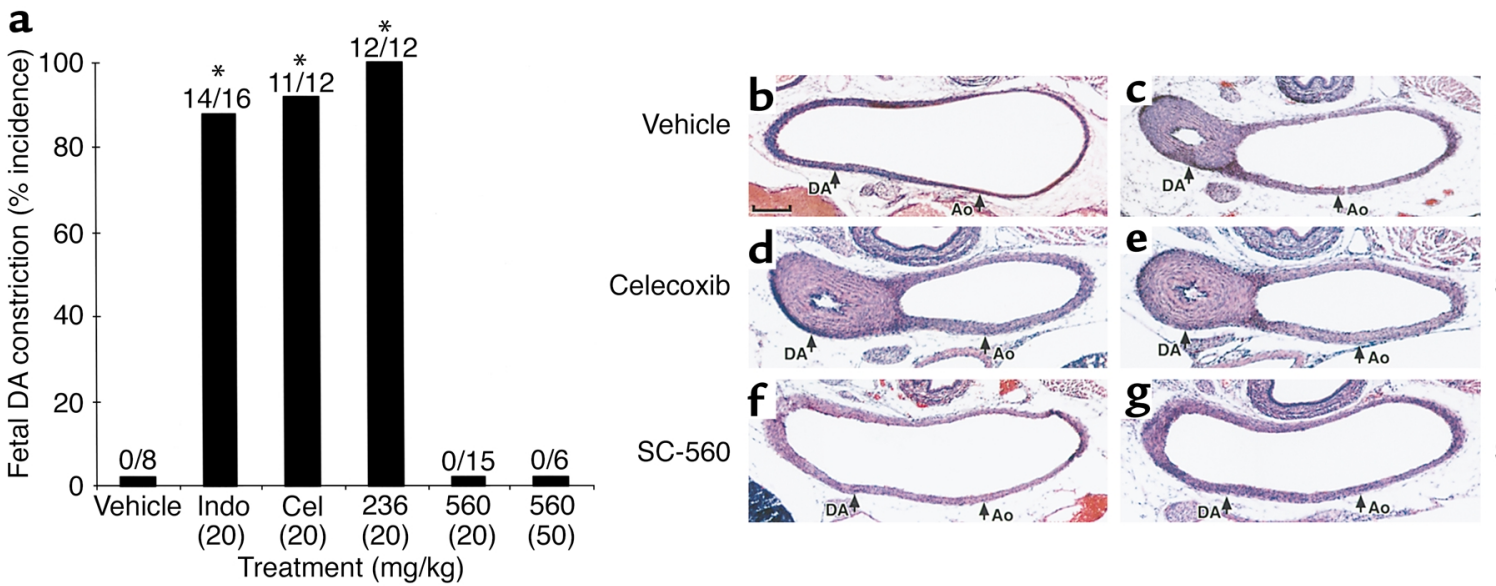

Indomethacin

\section{Figure 1}

Fetal DA constriction following acute isoform-selective or nonselective COX inhibition on gestation day 18. (a) Incidence (no. constricted/no. analyzed) of fetal DA constriction following treatment with COX inhibitors indomethacin (indo), celecoxib (cel), SC-236 (236), and SC-560 (560). *Significantly different from incidence in vehicle-treated mice. $P<0.01, n \geq 6$. (b-g) Histology of mouse DA after a single inhibitor treatment. (b) Vehicle. (c) Dual COX isoform inhibitor indomethacin, $20 \mathrm{mg} / \mathrm{kg}$. (d) COX-2-selective inhibitor celecoxib, 20 $\mathrm{mg} / \mathrm{kg}$. (e) COX-2-selective inhibitor SC-236, $20 \mathrm{mg} / \mathrm{kg}$. (f) COX-1-selective inhibitor SC-560, $20 \mathrm{mg} / \mathrm{kg}$. (g) SC-560, 50 mg/kg. Ao, descending aorta. Scale bar, $100 \mu \mathrm{m}$.

through gestation day 17 . For determining gestation lengths, pregnant females were monitored with digital cameras. Significant differences in average gestation lengths between vehicle and COX inhibitor treatments were determined by Student $t$ test.

Prostanoid treatments of neonatal mice. $\mathrm{COX}-1^{-1 /} / \mathrm{COX}-2^{-1-}$ pups were delivered by cesarean section after a normal gestation length of 18.5 days. For improved survival of COX $-1^{-/-} / C O X-2^{-/-}$pups, litters were maintained with pure oxygen for the first 30 minutes after birth. Litters were treated $0.5,1.5$, and 3.5 hours after birth with 0.5 $\mu \mathrm{g} / \mathrm{g}$ body weight of either $\mathrm{U} 46619, \mathrm{PGF}_{2 \alpha}$, sulprostone, carbaprostacyclin, or I-BOP, subcutaneously in 10 $\mu$ l saline (Cayman Chemical Co.), or with a single dose 0.5 hours after birth of ONO-AE1-329 $(0.5$ or $0.1 \mu \mathrm{g} / \mathrm{g}$ body weight) (kindly provided by Ono Pharmaceutical Co., Osaka, Japan). Pups were sacrificed 5 hours after birth for genotyping and DA analysis.

$P G E_{2}$ synthesis in fetal skin following maternal administration of COX inhibitors. $\mathrm{PGE}_{2}$ synthesis in excised fetal skin was measured to demonstrate whether SC-560 enters the fetus following maternal administration. SC-560 (50 $\mathrm{mg} / \mathrm{kg}$ oral gavage) or vehicle was administered twice a day on gestation days 15-17 and once on gestation day 18. Four hours after the last inhibitor dose, fetuses were delivered by cesarean section and euthanized by $4^{\circ} \mathrm{C}$ immersion. For the measurement of $\mathrm{PGE}_{2}$ synthesized from endogenous arachidonic acid, the entire skin from each fetus was homogenized in $1 \mathrm{ml}$ of PBS containing $100 \mu \mathrm{M}$ indomethacin. For measuring $\mathrm{PGE}_{2}$ synthesized from exogenous arachidonic acid, the entire skin from each fetus was homogenized in $1 \mathrm{ml}$ of PBS containing $50 \mu \mathrm{M}$ arachidonic acid, followed by a 30 -minute incubation at $37^{\circ} \mathrm{C}$. The homogenates from the experiments using endogenous or exogenous arachidonic acid were centrifuged at $12,000 \mathrm{~g}$ at $4^{\circ} \mathrm{C}$, and $\mathrm{PGE}_{2}$ concentrations in the supernatant were determined using a radioimmunoassay kit (Amersham Biosciences Corp., Piscataway, New Jersey, USA) according to the manufacturer's instructions. To determine whether SC-236 reduced PG production in tissue rich in $\mathrm{COX}-1, \mathrm{PGE}_{2}$ was measured in fetal skin homogenates following the administration of SC-236 $(20 \mathrm{mg} / \mathrm{kg}$ oral gavage) once a day on gestation days 15 and 17 , followed by $50 \mathrm{mg} / \mathrm{kg}$ on gestation day $18 . \mathrm{PGE}_{2}$ analysis was performed as described above for SC-560-treated mice.

\section{Results}

Acute inbibition of COX-2, but not COX-1, induces premature constriction of the fetal $D A$. To determine the relative contribution of the COX isoforms in maintaining DA

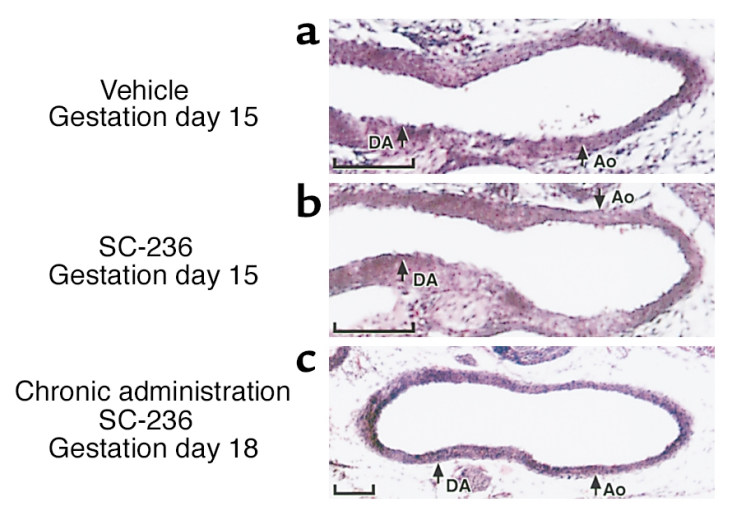

Figure 2

Chronic inhibition of COX-2 during gestation does not result in fetal DA constriction. Analysis of fetal DA on day 15 of gestation, 4 hours following administration of (a) vehicle or (b) COX-2-selective inhibitor SC-236. (c) Chronic administration (on gestation days 15-18) of SC-236 and fetal analysis 4 hours after the dose on gestation day 18 . Scale bar, $100 \mu \mathrm{m}$. 


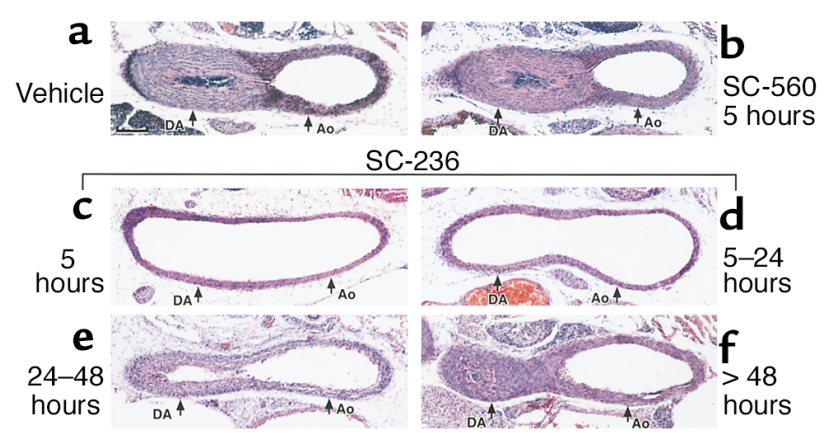

Figure 3

Neonatal patent DA following chronic COX-2 inhibition. Maternal administration of (a) vehicle, (b) COX-1-selective inhibitor SC-560, or (c-f) COX-2-selective inhibitor SC-236 on gestation days 15-18. Histological DA analysis of wild-type neonates sacrificed 5 hours after birth is shown in a-c. $\mathbf{d}-\mathbf{f}$ shows postmortem DA analysis of wild-type neonates that died (d) between 5 and 24 hours after birth, (e) between 24 and 48 hours after birth, or ( $\mathbf{f})$ were sacrificed after 48 hours for comparison. Scale bar, $100 \mu \mathrm{m}$.

dilation in utero, we analyzed the fetal mouse DA following a single maternal dose of selective inhibitors of COX-1 or COX-2 on gestation day 18 (Figure 1a). As expected, histological analysis demonstrated that the DA constricted in wild-type fetuses following indomethacin administration (Figure 1c), whereas the DA remained dilated following vehicle administration (Figure 1b). Similar to the effects of indomethacin, the COX-2-selective inhibitors celecoxib (Figure 1d) and SC-236 (Figure 1e) also induced DA constriction. In contrast to indomethacin and the COX-2 inhibitors, the COX-1-selective inhibitor SC-560 (22), at the equivalent dose (Figure 1f) as well as a 2.5 -fold higher dose (Figure 1g), failed to induce constriction of the fetal DA.

Chronic inhibition of COX-2 during gestation does not produce fetal DA constriction. In contrast to DA constriction induced by a single dose of COX-2 inhibitor SC-236 on gestation day 18 (Figure 1e), administration of SC-236 earlier in gestation (gestation day 15) did not produce an observable effect on the fetal DA (compare Figure 2a with Figure 2b). Furthermore, DA constriction was not induced by SC-236 treatment on gestation day 17 (data not shown) or gestation day 18 (Figure $2 \mathrm{c}$ ) in the fetuses of dams that were treated with the COX-2 inhibitor beginning on gestation day 15 . Therefore, fetal DA constriction was not observed following chronic inhibition of COX-2 on gestation days 15-18.

Chronic inbibition of COX-2 during gestation produces neonatal patent $D A$. With the observation that fetal DA constriction did not occur following chronic inhibition of COX-2 during pregnancy (Figure 2), we examined the effect of this maternal dosing regimen on closure of the neonatal DA. The DA was completely occluded at 5 hours after birth in all wild-type neonates treated with vehicle during gestation (Figure 3a). In contrast, chronic maternal administration (on gestation days 15-18) of SC-236 resulted in a postnatal patent DA incidence of
$59 \%$ in neonates wild-type for both COX-1 and COX-2 (Table 1 and Figure 3c). Similar to the effects of SC-236, maternal administration of celecoxib on gestation days 15-18 resulted in a 53\% incidence of patent DA in wildtype neonates at 5 hours after birth. Furthermore, the extent to which COX-2 inhibition increased patent DA incidence was influenced by the COX-1 genotype. For neonates heterozygous- or homozygous-null for COX-1 and wild-type for COX-2, the COX-2 inhibitor (SC-236) treatment resulted in a patent DA incidence of $70 \%$ and $100 \%$, respectively (Table 1 ). There was no significant increase in patent DA incidence in $\mathrm{COX}-1^{+/+} / \mathrm{COX}-2^{-/-}$neonates following COX-2 inhibitor (SC-236) treatment (Table 1), suggesting that SC-236 treatment did not inhibit COX-1. These data demonstrate that chronic administration of COX-2-selective inhibitors during gestation significantly increased the incidence of patent DA after birth.

In contrast to the COX-2-selective inhibitors, chronic administration (on gestation days 15-18) of the COX-1-selective inhibitor SC-560 failed to induce patent DA in neonates wild-type for both COX-1 and COX-2 (Figure 3b). The COX-1-selective inhibitor also failed to induce patent DA in all neonates wild-type for COX-2, irrespective of the COX-1 genotype (Table 1). We previously reported that for COX-2-deficient neonates, reduction of the gene copy number of COX-1 significantly increases patent DA incidence (16). In the present study, SC-560 treatment significantly increased the patent DA incidence in neonates deficient in COX-2. One-hundred percent of the neonates deficient in COX-2 and wild-type for COX-1 showed patent DA following COX-1 inhibitor treatment, compared with the $35 \%$ incidence observed in neonates of this genotype following vehicle treatment (Table 1). Therefore, in COX-2-deficient neonates, the chronic inhibition of COX-1 during gestation eliminated COX-1dependent DA closure; however, for all neonates that expressed COX-2, there was no increase in patent DA incidence following COX-1 inhibitor treatment.

Maternal administration of SC-560 inhibits the activity of COX-1 in the fetus. COX-2-selective and nonselective COX inhibitors readily cross the placenta and enter the

\section{Table 1}

Neonatal patent DA incidence following isoform-selective COX inhibition

Percentage of neonates with patent DA

Genotype

$\mathrm{COX}-1^{+/+} / \mathrm{COX}-2^{+/+}$

Vehicle COX-1 inhibitor COX-2 inhibitor

$\operatorname{COX}-1^{+/+} / \mathrm{COX}-2^{-/-}$

$\mathrm{COX}-1^{+/-} / \mathrm{COX}-2^{+/+}$

$\mathrm{COX}-1^{-/-} / \mathrm{COX}-2^{+/+}$

0
35
0
0

0

$100^{\mathrm{A}}$

0

0

39

$70^{\mathrm{A}}$

0

$100^{\mathrm{A}, \mathrm{B}}$

Maternal administration of vehicle, COX-1 inhibitor (SC-560), or COX-2 inhibitor (SC-236) on gestation days 15-18. Patent DA incidence was determined in neonates 5 hours after birth. ${ }^{\text {AS }}$ ignificantly different from vehicletreated. $P<0.01, n \geq 8$. ${ }^{B}$ Significantly different from wild-type treated with COX-2 inhibitor. $P<0.01, n \geq 8$. 
Table 2

Maternal administration of SC-560 inhibits the activity of COX-1 expressed in the fetus

\begin{tabular}{|c|c|c|}
\hline Maternal treatment & $\begin{array}{c}\text { Endogenous } \\
\text { arachidonic acid } \\
\text { (ng } \mathrm{PGE}_{2} / \mathrm{g} \text { tissue) }\end{array}$ & $\begin{array}{c}\text { Exogenous } \\
\text { arachidonic acid } \\
\left(\mathrm{ng} \mathrm{PGE}_{2} / \mathrm{g} \text { tissue }\right)\end{array}$ \\
\hline Vehicle & $11 \pm 2$ & $4,397 \pm 79$ \\
\hline COX-1 inhibitor SC-560 & $0.4 \pm 0.1^{\mathrm{A}}$ & $182 \pm 46^{B}$ \\
\hline COX-2 inhibitor SC-236 & $7 \pm 3$ & $4,445 \pm 98$ \\
\hline
\end{tabular}

$\mathrm{PGE}_{2}(\mathrm{ng} / \mathrm{g}$ tissue) in fetal skin homogenates with and without addition of exogenous arachidonic acid following maternal administration of vehicle, COX-1 inhibitor, or COX-2 inhibitor on gestation days $15-18 . \mathrm{PGE}_{2}$ synthesis from exogenous arachidonic acid indicates fetal rather than maternal COX activity. Mean $\mathrm{PGE}_{2}$ level is shown $\pm \mathrm{SD}$. ASignificantly different from vehicle treatment with endogenous arachidonic acid. $P<0.01, n=5$. BSignificantly different from vehicle treatment with exogenous arachidonic acid. $P<0.01, n=5$.

fetal circulation (23-25). However, transfer of the COX-1-selective inhibitor SC-560 to the fetal circulation following maternal administration has not previously been reported. Therefore, to demonstrate that SC-560 crossed the placenta and entered the fetus following maternal administration, we measured $\mathrm{PGE}_{2}$ synthesis in fetal skin, which is a rich source of COX-1 (16). As shown in Table 2, maternal administration of SC-560 on gestation days 15-18 reduced the fetal skin concentration of $\mathrm{PGE}_{2}$ synthesized from endogenous arachidonic acid by more than 95\%. Because this observed reduction in fetal $\mathrm{PGE}_{2}$ may result from inhibiting the synthesis of $\mathrm{PGE}_{2}$ that is supplied to the fetus from maternal tissue, the inhibition of fetal COX-1 activity was confirmed by measuring $\mathrm{PGE}_{2}$ levels after the exogenous addition of arachidonic acid to fetal skin homogenates. Maternal administration of SC-560 on gestation days $15-18$ inhibited the synthesis of $\mathrm{PGE}_{2}$ formed from exogenous arachidonic acid by more than $95 \%$ (Table 2 ). Therefore, the maternal administration of SC-560 effectively inhibited the activity of COX-1 expressed in the fetus. In contrast to COX-1-selective inhibition, maternal administration of the COX-2-selective inhibitor SC-236 did not significantly reduce $\mathrm{PGE}_{2}$ levels in fetal skin homogenates (Table 2). Thus, COX-2 does not significantly contribute to $\mathrm{PGE}_{2}$ synthesis in fetal skin, and at the doses used, SC-236 does not inhibit COX-1.

Chronic inbibition of $\mathrm{COX}-2$ during gestation increases neonatal mortality. We previously reported that patent DA resulting from the genetic deficiency of COX-2 increases neonatal mortality (16). Because COX-2 inhibitor administration also increased the incidence of patent DA (Table 1), neonatal mortality was examined following COX-2 inhibition during gestation. More than $98 \%$ of the wild-type neonates, from dams treated with SC-236 on gestation days 15-18, were born alive after a normal gestation length. However, $71 \%$ of these neonates died during the first 48 hours following birth, compared with $2 \%$ of neonates from vehicle-treated dams. In the mice that survived beyond 48 hours following COX-2 inhibitor treat-

ment during gestation, there was no further increase in mortality when observed to the age of weaning. In neonates deficient in COX-1 and wild-type for COX-2, COX-2 inhibitor treatment resulted in a $100 \%$ incidence of neonatal mortality. Therefore, chronic inhibition of COX-2 during gestation significantly increased neonatal mortality.

Postmortem analysis was performed to identify pathologies responsible for the observed mortality in neonates born to dams chronically treated with a COX-2 inhibitor (on gestation days 15-18). Lungs, heart, kidney, liver, pancreas, spleen, thymus, intestine, and stomach of neonates from dams treated with SC-236 were indistinguishable in histological analyses from vehicle-treated controls. The only pathology observed in neonates following chronic COX-2 inhibitor treatment during gestation was patent DA.

Considerable variability in the duration of neonatal survival was observed following chronic treatment with SC-236 during gestation. To determine whether variability in DA closure could explain the differences in survival duration, the extent of DA closure was compared with survival duration for neonates that died at different times during the first 48 hours after birth. Histological analysis of the DA from neonates that died during the first 24 hours after birth shows no evidence of closure (Figure $3 \mathrm{~d}$ ). Partial closure of the DA with narrowing of the DA lumen was evident in neonates that died from 24 to 48 hours after birth (Figure 3e), as compared with the completely occluded DA of neonates that survived longer than 48 hours and were sacrificed (Figure 3f). Therefore, partial closure of the DA correlated with prolonged survival up to 48 hours, whereas the complete failure of DA closure correlated with mortality during the first 24 hours after birth.

Selective inbibition of $C O X-1$ during pregnancy delays the onset offull-term and preterm labor. In humans, the therapeutic usefulness of dual COX isoform inhibitors for prolonging gestation is limited, in part, by the adverse effects of fetal DA constriction or postnatal patent DA $(4-7,11,12)$. After finding that these adverse effects in wild-type mice did not occur with SC-560, we examined

\section{Table 3}

Inhibition of COX-1 delays the initiation of full-term and preterm labor

\section{Treatment}

Vehicle

COX-1 inhibitor SC-560

COX-2 inhibitor celecoxib

COX-2 inhibitor SC-236

Vehicle + LPS

SC $-560+$ LPS

Mean gestation length (days)
$18.7 \pm 0.2$
$20.7 \pm 0.3^{\mathrm{A}}$
$18.8 \pm 0.2$
$18.5 \pm 0.2$
$15.7 \pm 0.2$
$19.1 \pm 1.4^{\mathrm{B}}$

Vehicle, COX-1 inhibitor, or COX-2 inhibitors were administered to wild type dams on gestation days 15-18 or gestation days 14-17 (LPS-treated dams). Mean gestation length is shown \pm SD. ASignificantly different from gestation lengths of mice treated with vehicle or COX-2-selective inhibitor. $P<0.01, n \geq 8$. BSignificantly different from gestation length of vehicle-treated mice following LPS-induced labor. $P<0.01, n=8$. 
Table 4

Effects of exogenous prostanoids on neonatal DA closure

\begin{tabular}{lc} 
Treatment & $\begin{array}{c}\text { Induced DA closure } \\
\text { (\% incidence) }\end{array}$ \\
Vehicle & $0(0 / 15)$ \\
PGF $_{2 \alpha}$ & $0(0 / 6)$ \\
Sulprostone & $0(0 / 6)$ \\
Carbaprostacyclin & $0(0 / 8)$ \\
ONO-AE1-329 & $0(0 / 6)$ \\
U46619 & $100(12 / 12)$ \\
I-BOP & $100(7 / 7)$ \\
\hline
\end{tabular}

Percent incidence (no. mice with closed DA/no. mice analyzed) of DA closure in $\mathrm{COX}_{-1} 1^{-1-} / \mathrm{COX}_{-2} 2^{-/-}$neonates following prostanoid treatments.

the effectiveness of this COX-1-selective inhibitor for delaying the initiation of full-term labor in mice. Treatment with SC-560 beginning on gestation day 15 resulted in an average gestation length of 20.7 days, whereas the average gestation length of vehicle-treated mice was 18.7 days (Table 3). Although the COX-1 inhibitor prolonged the length of gestation, the duration of labor was not significantly different from that of vehicle-treated mice (data not shown). In contrast to COX-1 inhibition, neither of the COX-2-selective inhibitors, celecoxib and SC-236, significantly affected the length of gestation (Table 3 ).

The tocolytic effects of COX-1-selective inhibition were also analyzed using a model of preterm labor induced by LPS $(20,21)$. Intraperitoneal injection of LPS to six pregnant mice on gestation day 15 initiated preterm labor within 24 hours in all six, and resulted in a $100 \%$ incidence of fetal death. However, when eight mice were treated with SC-560 on gestation days 14-17, none of them littered during the first 24 hours after LPS treatment. SC-560 treatment significantly prolonged the average gestation length of LPS-treated mice (Table 3 ) and increased the number of litters that were carried to term (six of eight). SC-560 treatment also significantly increased overall litter survival from zero to $72 \%$ following LPS treatment. These findings indicate that inhibition of COX-1 effectively delays the initiation of full-term and preterm labor in mice.

Thromboxane receptor agonists induce neonatal closure of the DA. The deficiency of both COX-1 and COX-2 in mice results in a $100 \%$ incidence of neonatal patent DA (16) and provides a model for identifying prostanoids with activity for initiating closure of the DA after birth. Following treatment of $\mathrm{COX}-1^{-/-} / \mathrm{COX}-2^{-/-}$neonates with vasoactive prostanoids or stable prostanoid analogues, the DA was examined histologically for evidence of DA closure (Table 4). Neither PGF $2 \alpha$ nor sulprostone (an EP1/EP3 agonist), prostanoids previously shown to produce constriction in the vasculature (26, $27)$, induced closure of the DA when administered to COX $-1^{-/-} / \mathrm{COX}-2^{-/-}$neonates. The stable prostacyclin analogue, carbaprostacyclin, also failed to induce closure of the DA. Although the treatment of neonatal mice with $\mathrm{PGE}_{2}$ dilates the DA after birth (28), mice deficient in the $\mathrm{EP}_{4}$ type $\mathrm{PGE}_{2}$ receptor show increased patent DA incidence $(28,29)$, suggesting a role for the $\mathrm{EP}_{4}$ receptor in DA closure. Therefore we treated COX-1 $1^{-/-} / \mathrm{COX}-2^{-/-}$neonates with the $\mathrm{EP}_{4}$-selective agonist ONO-AE1-329 (30) to determine the potential for $\mathrm{EP}_{4}$-selective activation to induce $\mathrm{DA}$ closure. The $\mathrm{EP}_{4}$ receptor agonist not only failed to induce DA closure in $\mathrm{COX}-1^{-1-} / \mathrm{COX}-2^{-/-}$neonates, but also prevented closure in wild-type neonates (Figure 4a), similar to the reported effect of $\mathrm{PGE}_{2}(28,31)$. In contrast to the other prostanoids, the $\mathrm{PGH}_{2}$ analogue $\mathrm{U} 46619$ (32) and the thromboxane $\mathrm{A}_{2}$ analogue I-BOP (33), two structurally distinct thromboxane receptor agonists, both induced DA closure (Table 4 and Figure $4 \mathrm{~b}$ ) that was histologically indistinguishable from that of vehicle-treated wild-type neonates at 5 hours after birth (Figure 4a).

\section{Discussion}

Despite the availability of several classes of pharmacological treatments to delay preterm delivery, premature birth remains the leading cause of neonatal mortality and morbidity. Currently available tocolytics, including COX inhibitors such as indomethacin, have been shown to be effective in delaying labor in the short term (48-72 hours), but their efficacy for the long-term tocolytic maintenance necessary to significantly improve neonatal health has not been demonstrated $(1-3,34)$. Therefore there is considerable interest in identifying novel medications for safe and effective tocolysis.

The tocolytic effects of nonselective COX inhibitors in humans may result from inhibiting COX-1 and/or COX-2. In mice, the initiation of labor results from the reduction of serum progesterone following $\mathrm{PGF}_{2 \alpha^{-}}$ induced lysis of the corpus luteum (35). In COX-1-deficient mice, the reduced synthesis of $\mathrm{PGF}_{2 \alpha}$ delays the initiation of labor (36), whereas the effects of COX-2 deficiency on the initiation of labor are not known because of female infertility $(37,38)$. We studied the a

\footnotetext{
Figure 4

Prostanoid analogues modulate neonatal DA closure. (a) COX $-1^{+/+} / \mathrm{COX}-2^{+/+}$neonates treated with vehicle (top row) or $\mathrm{EP}_{4}$-selective agonist ONO-AE1-329 (bottom row). (b) $\mathrm{COX}-1^{-/-} / \mathrm{COX}-2^{-/-}$neonates treated with vehicle (top row) or $\mathrm{PGH}_{2}$ analogue $\mathrm{U} 46619$ (bottom row). Scale bar, $100 \mu \mathrm{m}$.
}

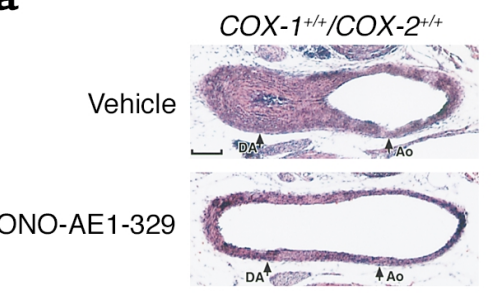

b

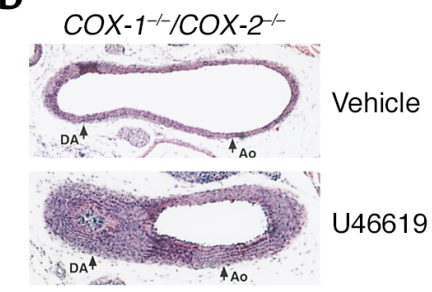


functions of the two COX isoforms in the initiation of full-term labor in mice by comparing tocolytic effects of selective inhibition of either COX-1 or COX-2. The treatment of pregnant mice with a selective inhibitor of COX-1 delayed the initiation of full-term labor, whereas COX-2-selective inhibitors produced no effect on gestation length (Table 3). Recently, COX-2 inhibition in mice has been shown to delay the initiation of LPSinduced preterm labor $(20,21)$. However, the present study indicates that COX-2-selective inhibition has adverse effects on the fetal or neonatal DA that may compromise neonatal health. With our findings that COX-1 inhibition did not produce these adverse effects and significantly delayed the initiation of full-term labor, we examined the tocolytic effectiveness of COX-1 inhibition on preterm labor induced by LPS. COX-1-selective inhibition prolonged the average gestation length following LPS treatment (Table 3), which significantly improved neonatal survival. Therefore, in mice, PG synthesis dependent on the activity of COX-1 contributes to the initiation of full-term labor and LPS-induced preterm labor.

COX inhibitors have been used for decades to delay preterm delivery, but their usefulness is limited, in part, by constriction of the fetal DA. Human and mouse fetuses are highly sensitive to the DA constrictor effects of indomethacin $(6,16)$, and recently, COX-2-selective inhibitors were shown to constrict the fetal DA in mice and sheep $(23,39)$. In the present study, we determined the DA-constricting effects of COX isoform-selective and-nonselective inhibition in the mouse fetus. When administered near the end of gestation, the COX-2selective inhibitors SC-236 and celecoxib both induced fetal DA constriction similar to that resulting from indomethacin treatment (Figure 1). In contrast, the COX-1-selective inhibitor SC-560 failed to induce constriction of the fetal DA (Figure 1). Thus, in mice, the adverse effect of indomethacin-induced fetal DA constriction following acute maternal administration results from inhibiting the synthesis of PGs derived primarily via COX-2 rather than COX-1.

Recently, we and others described the failure of DA closure in neonatal mice deficient in both COX-1 and COX-2 $(16,39)$, and our studies provided the initial insight into the key role of COX-2 in the normal closure of the DA (16). In contrast to a report by Reese et al. (39), our findings (16) demonstrated that high levels of COX-2 were expressed in the smooth muscle cells of the DA during closure of the vessel after birth, suggesting that COX-2 expressed in the DA functions in the closure of the vessel. Because COX-2-selective inhibitors (and nonselective COX inhibitors) are known to cross the placenta (23-25), we hypothesized that following chronic maternal administration, COX-2-selective inhibitors may enter the fetal circulation and inhibit COX-2 expressed in the DA, thereby preventing normal closure in the neonate. In support of this hypothesis, our present findings indicate that in wild-type mice, chronic inhibition of
COX-2, but not COX-1, significantly increased the incidence of patent DA following birth (Table 1).

In the present study, we observed that neonatal patent DA resulted from chronic inhibition of COX-2 during gestation, whereas acute inhibition of COX-2 late in gestation produced fetal DA constriction. The contrasting effects of COX-2 inhibition suggest opposing actions of different prostanoids on the regulation of DA tone. $\mathrm{PGE}_{2}$ in the fetal circulation, supplied in part by the placenta, maintains a dilated DA by attenuating DA muscle contraction $(9,40)$. This suggests that constriction of the fetal DA following acute COX-2 inhibition late in gestation results from inhibiting the synthesis of $\mathrm{PGE}_{2}$ supplied to the DA by the placenta. Our previous findings demonstrate that the DA, unlike the adjacent aorta or pulmonary artery, expresses a significant level of COX-2 following birth (16), which we propose is responsible for the synthesis of prostanoids that contribute to DA muscle contraction. This is consistent with our finding that stable analogues of $\mathrm{PGH}_{2}$ and thromboxane $\mathrm{A}_{2}$ both induced DA

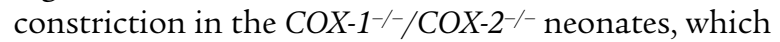
without intervention show a $100 \%$ incidence of patent DA (Table 4 and Figure 4). Previous studies in sheep determined that thromboxane $\mathrm{A}_{2}$ is not synthesized by DA tissue $(41,42)$, and these data in combination with our present findings suggest a predominant role for the known vasoconstrictor $\mathrm{PGH}_{2}$ (43) as the PG contributing to DA closure. Therefore, chronic administration of COX-2 inhibitors during gestation may sufficiently inhibit the synthesis of $\mathrm{PGH}_{2}$ produced by COX-2 in the DA to attenuate muscle contraction and result in patent DA.

The incidence and clinical severity of patent DA is significantly increased in infants born to women treated with indomethacin $(4,11,12)$. Recently, indomethacininduced ovine fetal DA constriction was shown to damage DA smooth muscle and make the DA refractory to postnatal DA constriction (44). This suggests that fetal DA constriction may contribute to postnatal patent DA. However, we observed that acute COX-2 inhibitor treatment on gestation day 15 did not constrict the fetal DA (Figure 2), suggesting that similar to reported effects of indomethacin $(7,8)$, incomplete development of DA contractile function in preterm fetuses makes the DA resistant to COX-2 inhibitor-induced constriction. Furthermore, chronic administration of the COX-2 inhibitor beginning on gestation day 15 produced resistance to SC-236induced constriction on gestation day 18 (Figure 2). Therefore, our data indicate that fetal DA constriction does not appear to be required for the neonatal patent DA that we observed following COX-2 inhibitor treatment during gestation.

Although our findings indicate that patent DA did not result from the inhibition of COX-1 in wild-type mice, a role for COX-1 in postnatal DA closure was identified by combining pharmacological inhibition with genetic inactivation. The spontaneous patent DA 
incidence in COX-2-deficient mice (35\%) was increased to $100 \%$ following COX-1 inhibitor treatment during pregnancy (Table 1). The COX-1 inhibitor produced patent DA only in mice genetically deficient in COX-2, whereas patent DA resulting from COX-2 inhibition did not require the genetic inactivation of COX-1. Thus, COX-1 plays only a compensatory role, and COX-2 is the isoform primarily responsible for DA closure.

Chronic inhibition of COX-2 during gestation also significantly increased neonatal mortality during the first 48 hours following birth. Analysis of the pups that died demonstrated that the DA had failed to close, whereas patent DA was not observed in neonates that survived beyond 48 hours after birth (Figure 3 ). In addition to the regulation of DA tone, PGs, particularly $\mathrm{PGI}_{2}$, are also believed to be important for dilation of the pulmonary vasculature following birth $(45,46)$. It is possible that by inhibiting $\mathrm{PGI}_{2}$ synthesis, $\mathrm{COX}$ inhibitors may increase pulmonary vascular resistance, which may impede normal postnatal DA closure. However, analysis of the lungs of neonates that died following COX-2 inhibitor treatment during gestation failed to find histological evidence of pulmonary hypertension such as pulmonary vascular wall remodeling. Furthermore, a stable $\mathrm{PGI}_{2}$ analogue was not effective for inducing postnatal closure of the DA (Table 4 and Figure 4), suggesting that pulmonary hypertension was not responsible for the observed patent DA following COX-2-selective inhibition. Although other unidentified pathologies may occur, the correlation of patent DA incidence and severity with neonatal mortality and survival duration, respectively, suggests that patent DA directly contributed to COX-2 inhibitor-induced neonatal mortality.

In the current study we have demonstrated that COX-1-selective inhibition, but not COX-2-selective inhibition, effectively delays labor without adverse DA effects in the fetus or neonate. Recently, Reese et al. described the effects of COX-2 inhibitor treatment during pregnancy on the length of full-term gestation and neonatal survival in mice (39). Similar to our current findings, the report by Reese et al. showed that the normal gestation length is not prolonged by a dose of celecoxib selective for the inhibition of COX-2 (10 $\mathrm{mg} / \mathrm{kg}$ ); however, gestation length was prolonged by treatment with celecoxib at $600 \mathrm{mg} / \mathrm{kg}$, a dose that the authors suggested may inhibit both COX-1 and COX-2 (39). Our current findings of prolonged gestation following COX-1-selective inhibition suggest that the prolonged gestation described by Reese et al. following treatment with high doses of celecoxib may have resulted primarily from inhibiting COX-1. Prolonged gestation in mice, resulting from either COX-1 deficiency $(17,36,39)$ or from celecoxib doses that may also inhibit COX-1 (39), is associated with reduced pup survival. However, Reese et al. also reported increased pup mortality with doses of celecoxib $(200 \mathrm{mg} / \mathrm{kg})$ that did not significantly prolong gestation (39), although the cause or causes of mortality were not identified. Our current findings of patent DA and resulting neonatal mortality following chronic COX-2 inhibition during pregnancy suggest that the increased pup mortality observed by Reese et al. following celecoxib treatment may have resulted in part from patent DA. Overall, the results to date support our conclusion that COX-1-selective inhibition may provide a novel method of delaying labor.

Premature birth significantly increases the incidence of patent DA, suggesting that premature infants may be especially susceptible to medications that prevent or delay closure of the DA after birth. Because women in premature labor are the target population for tocolysis, particular caution is warranted for novel tocolytics with the potential to attenuate DA closure in the newborn. Recently, numerous reports have suggested that COX-2-selective inhibitors may be effective for delaying preterm birth in women $(2,14,15,20,47,48)$. Our previous genetic findings, together with the present pharmacological studies, indicate that in mice, COX-2 activity in the fetus or neonate is important for closure of the DA after birth. If the mechanisms of DA closure are conserved between species, the use of COX-2-selective inhibitors as tocolytics in women may impede normal closure of the DA after birth. Furthermore, our findings suggest that COX-1-selective inhibitors may provide effective tocolysis without the adverse DA effects of nonselective or COX-2-selective inhibitors.

\section{Acknowledgments}

We thank Ono Pharmaceutical Co., Osaka, Japan, for the generous gift of ONO-AE1-329.

1. Bernal, A. 2001. Overview of current research in parturition. Exp. Physi ol. 86:213-222.

2. Vause, S., and Johnston, T. 2000. Management of preterm labour. Arch. Dis. Child. Fetal Neonatal Ed. 83:F79-F85.

3. Norwitz, E.R., Robinson, J.N., and Challis, J.R. 1999. The control of labor. N. Engl. J. Med. 341:660-666.

4. Norton, M.E. 1997. Teratogen update: fetal effects of indomethacin administration during pregnancy. Teratology. 56:282-292.

5. Moise, K.J.J., et al. 1988. Indomethacin in the treatment of premature labor. Effects on the fetal ductus arteriosus. N. Engl. J. Med. 319:327-331.

6. Vermillion, S.T., Scardo, J.A., Lashus, A.G., and Wiles, H.B. 1997. The effect of indomethacin tocolysis on fetal ductus arteriosus constriction with advancing gestational age. Am. J. Obstet. Gynecol. 177:256-259.

7. Moise, K.J.J. 1993. Effect of advancing gestational age on the frequency of fetal ductal constriction in association with maternal indomethacin use. Am. J. Obstet. Gynecol. 168:1350-1353.

8. Momma, K., and Takao, A. 1987. In vivo constriction of the ductus arteriosus by nonsteroidal antiinflammatory drugs in near-term and preterm fetal rats. Pediatr. Res. 22:567-572.

9. Smith, G.C.S. 1998. The pharmacology of the ductus arteriosus. Pharmacol. Rev. 50:35-58.

10. Eronen, M. 1993. The hemodynamic effects of antenatal indomethacin and a beta-sympathomimetic agent on the fetus and the newborn: a randomized study. Pediatr. Res. 33:615-619.

11. Hammerman, C., et al. 1998. Indomethacin tocolysis increases postnatal patent ductus arteriosus severity. Pediatrics. 102:E561-E564.

12. Norton, M.E., Merrill, J., Cooper, B.A., Kuller, J.A., and Clyman, R.I. 1993. Neonatal complications after the administration of indomethacin for preterm labor. N. Engl. J. Med. 329:1602-1607.

13. Hammerman, C., and Kaplan, M. 1999. Patent ductus arteriousus in the premature neonate: current concepts in pharmacological management. Paediatr. Drugs. 1:81-92.

14. Sadovsky, Y., et al. 2000. Effective diminution of amniotic prostaglandin production by selective inhibitors of cyclooxygenase type 2. Am. J. Obstet. Gynecol. 182:370-376. 
15. Vane, J.R., and Botting, R.M. 1998. Anti-inflammatory drugs and their mechanism of action. Inflamm. Res. 47(Suppl. 2):S78-S87.

16. Loftin, C.D., et al. 2001. Failure of ductus arteriosus closure and remodeling in neonatal mice deficient in cyclooxygenase- 1 and cyclooxygenase2. Proc. Natl. Acad. Sci. USA. 98:1059-1064.

17. Langenbach, R., et al. 1995. Prostaglandin synthase 1 gene disruption in mice reduces arachidonic acid-induced inflammation and indomethacin-induced gastric ulceration. Cell. 83:483-492.

18. Morham, S.G., et al. 1995. Prostaglandin synthase 2 gene disruption causes severe renal pathology in the mouse. Cell. 83:473-482.

19. Penning, T.D., et al. 1997. Synthesis and biological evaluation of the 1,5diarylpyrazole class of cyclooxygenase- 2 inhibitors: identification of 4 [5-(4-methylphenyl)-3-(trifluoromethyl)-1H-pyrazol-1-yl]benze nesulfonamide (SC-58635, celecoxib). J. Med. Chem. 40:1347-1365.

20. Sakai, M., et al. 2001. Evaluation of the tocolytic effect of a selective cyclooxygenase- 2 inhibitor in a mouse model of lipopolysaccharideinduced preterm delivery. Mol. Hum. Reprod. 6:595-602.

21. Gross, G., et al. 2000. Inhibition of cyclooxygenase-2 prevents inflammation-mediated preterm labor in the mouse. Amer. J. Physiol. Regul. Integr. Comp. Physiol. 278:R1415-R1423.

22. Smith, C.J., et al. 1998. Pharmacological analysis of cyclooxygenase-1 in inflammation. Proc. Natl. Acad. Sci. USA. 95:13313-13318.

23. Takahashi, Y., et al. 2000. Cyclooxygenase- 2 inhibitors constrict the fetal lamb ductus arteriosus both in vitro and in vivo. Amer. J. Physiol. Regul. Integr. Comp. Physiol. 278:R1496-R1505.

24. Moise, K.J.J., et al. 1990. Placental transfer of indomethacin in the human pregnancy. Am. J. Obstet. Gynecol. 162:549-554.

25. Kramer, W.B., et al. 1995. Placental transfer of sulindac and its active sulfide metabolite in humans. Am. J. Obstet. Gynecol. 172:886-890.

26. Smith, G.C., and McGrath, J.C. 1995. Contractile effects of prostanoids on fetal rabbit ductus arteriosus. J. Cardiovasc. Pharmacol. 25:113-118.

27. Lock, J.E., Olley, P.M., and Coceani, F. 1980. Direct pulmonary vascular responses to prostaglandins in the conscious newborn lamb. Am. J. Physiol. 238:H631-H638.

28. Nguyen, M., et al. 1997. The prostaglandin receptor EP4 triggers remodelling of the cardiovascular system at birth. Nature. 390:78-81.

29. Segi, E., et al. 1998. Patent ductus arteriosus and neonatal death in prostaglandin receptor EP4-deficient mice. Biochem. Biophys. Res. Commun. 246:7-12.

30. Suzawa, T., et al. 2000. The role of prostaglandin E receptor subtypes (EP1, EP2, EP3, and EP4) in bone resorption: an analysis using specific agonists for the respective EPs. Endocrinology. 141:1554-1559.

31. Takizawa, T., et al. 1995. Dilating effect of prostaglandin on the constricted ductus arteriosus in newborn rats. J. Toxicol. Sci. 20:29-32.

32. Kattelman, E.J., Venton, D.L., and Le Breton, G.C. 1986. Characterization of U46619 binding in unactivated, intact human platelets and determination of binding site affinities of four TXA2/PGH2 receptor antagonists (13-APA, BM 13.177, ONO 3708 and SQ 29,548). Thromb. Res. 41:471-481.

33. Morinelli, T.A., et al. 1989. Characterization of an 125I-labeled thromboxane A2/prostaglandin $\mathrm{H} 2$ receptor agonist. J. Pharmacol. Exp. Ther. 251:557-562.

34. Thornton, S., Vatish, M., and Slater, D. 2001. Oxytocin antagonists: clinical and scientific considerations. Exp. Physiol. 86:297-302.

35. Sugimoto, Y., et al. 1997. Failure of parturition in mice lacking the prostaglandin F receptor. Science. 277:681-683.

36. Gross, G.A., et al. 1998. Opposing actions of prostaglandins and oxytocin determine the onset of murine labor. Proc. Natl. Acad. Sci. USA. 95:11875-11879.

37. Lim, H., et al. 1997. Multiple female reproductive failures in cyclooxygenase 2-deficient mice. Cell. 91:197-208.

38. Dinchuk, J.E., et al. 1995. Renal abnormalities and an altered inflammatory response in mice lacking cyclooxygenase II. Nature. 378:406-409.

39. Reese, J., et al. 2000. Coordinated regulation of fetal and maternal prostaglandins directs successful birth and postnatal adaptation in the mouse. Proc. Natl. Acad. Sci. USA. 97:9759-9764.

40. Thorburn, G.D. 1992. The placenta, PGE2 and parturition. Early Hum. Dev. 29:63-73.

41. Pace-Asciak, C.R., and Rangaraj, G. 1983. Application of prostaglandinprofiling techniques to study the release of prostaglandins from the fetal lamb ductus arteriosus. Biochim. Biophys. Acta. 750:330-333.

42. Pace-Asciak, C.R., and Rangaraj, G. 1983. Release of prostaglandins from intact fetal lamb ductus arteriosus. Can. J. Biochem. Cell Biol. 61:240-247.

43. Lin, L., Balazy, M., Pagano, P.J., and Nasjletti, A. 1994. Expression of prostaglandin $\mathrm{H} 2$-mediated mechanism of vascular contraction in hypertensive rats. Relation to lipoxygenase and prostacyclin synthase activities. Circ. Res. 74:197-205.

44. Clyman, R.I., et al. 2001. In utero remodeling of the fetal lamb ductus arteriosus: the role of antenatal indomethacin and avascular zone thickness on vasa vasorum proliferation, neointima formation, and cell death. Circulation. 103:1806-1812.

45. Shaul, P.W. 1999. Regulation of vasodilator synthesis during lung development. Early Hum. Dev. 54:271-294.

46. Davidge, S.T. 2001. Prostaglandin $H$ synthase and vascular function. Circ. Res. 89:650-660.

47. Sawdy, R.J., Slater, D.M., Dennes, W.J.B., Sullivan, M.H.F., and Bennett, P.R. 2000. The roles of the cyclo-oxygenases types one and two in prostaglandin synthesis in human fetal membranes at term. Placenta. 21:54-57.

48. Yousif, M.H., and Thulesius, O. 1998. Tocolytic effect of the cyclooxygenase-2 inhibitor, meloxicam: studies on uterine contractions in the rat. J. Pharm. Pharmacol. 50:681-685. 